\title{
Stents liberadores de medicamentos en enfermedad coronaria multivaso (dos y tres vasos)
}

\section{Diego Velásquez}

\author{
CES Cardiología - Universidad CES, Medellín, Colombia
}

Recibido el 4 de abril de 2017; aceptado el 10 de noviembre de 2017

Disponible en Internet el 24 de diciembre de 2017

\section{PALABRAS CLAVE}

Stents liberadores de medicamento;

Enfermedad

coronaria multivaso;

Revascularización

coronaria

\section{KEYWORDS}

Drug-eluting stents; Multivessel coronary artery; Coronary revascularization

\begin{abstract}
Resumen Los stents liberadores de medicamento (DES, su sigla en inglés por drug-eluting stents) han disminuido de manera significativa la reestenosis clínica en comparación con los stents no medicados (BMS, del inglés bare-metal stents). Por otra parte, los cambios realizados a los DES de nueva generación han llevado a una disminución adicional en la necesidad de revascularización repetida y en las tasas de trombosis respecto a los DES de primera generación. Esta mejoría en la seguridad y eficacia en los DES de segunda generación, debería llevar a mejores resultados en la intervención coronaria percutánea, pero a la fecha la mayor parte de la evidencia disponible respecto a la revascularización en el subgrupo de pacientes con enfermedad coronaria multivaso (dos y tres vasos) corresponde a angioplastia con balón, BMS y DES de primera generación. Se presenta la evidencia disponible y se hacen las recomendaciones con base en la misma.

(C) 2017 Publicado por Elsevier España, S.L.U. en nombre de Sociedad Colombiana de Cardiología y Cirugía Cardiovascular. Este es un artículo Open Access bajo la licencia CC BY-NC-ND (http:// creativecommons.org/licenses/by-nc-nd/4.0/).
\end{abstract}

\section{Drug-eluting stents in multivessel coronary disease (two and three vessels)}

Abstract Drug-eluting stents (DES) have significantly decreased clinical restenosis compared with bare metal stents (BMS). Furthermore, the changes made in the latest-generation DESs have led to an additional reduction in the need for repeat revascularization, and in thrombosis rates, with respect to first-generation DESs. This improvement in the safety and efficacy of secondgeneration DESs should lead to better results in percutaneous coronary interventions, but to date, most of the available evidence with regard to revascularization in the subgroup of patients

Correo electrónico: elidieg057@gmail.com 
with multivessel coronary disease (two and three vessels) comes from balloon angioplasty with BMSs and first generation DESs. The available evidence is presented, and recommendations made based on this evidence.

(c) 2017 Published by Elsevier España, S.L.U. on behalf of Sociedad Colombiana de Cardiología y Cirugía Cardiovascular. This is an open access article under the CC BY-NC-ND license (http: / / creativecommons.org/licenses/by-nc-nd/4.0/).

\section{Introducción}

Los stents liberadores de medicamento (DES, su sigla en inglés por drug-eluting stents) disminuyeron de manera significativa la reestenosis clínica en comparación con los stents no medicados (BMS, del inglés bare-metal stents) $)^{1-4}$. No obstante, los estudios a largo plazo documentaron una propensión de los DES de primera generación Cypher ${ }^{\circledR}$ (stent liberador de sirolimus, SES su sigla en inglés por sirolimuseluting stent) y Taxus ${ }^{\circledR}$ (stent liberador de paclitaxel, PES su sigla en inglés por paclitaxel-eluting stents) a trombosis muy tardía ${ }^{5-8}$. Las nuevas generaciones de los DES se han caracterizado por tener una estructura con puntales (struts) más delgados y plataformas metálicas que liberan drogas antiproliferativas basadas en limus, desde (i) polímeros durables que tienen una mejor biocompatibilidad y una menor masa polimérica, (ii) polímeros biodegradables, o (iii) superficies libres de polímero. Estos cambios en los DES de segunda generación han llevado a una disminución adicional en la necesidad de nueva revascularización, de hasta un $10 \%$ a $20 \%$ en comparación con los DES de primera generación y a una mejoría en la seguridad, traducida en menor incidencia de trombosis probable o definida de aproximadamente un $50 \%$, particularmente durante el seguimiento tardío, y de muerte e infarto de miocardio ${ }^{9-14}$. Esta mejoría en la seguridad y eficacia con los DES de segunda generación debería llevar a mejores resultados en la intervención coronaria percutánea (ICP) en enfermedad de dos y tres vasos. Sin embargo, buena parte de la evidencia de revascularización en este subgrupo de pacientes con enfermedad de dos o tres vasos es con POBA (sigla en inglés por plain old balloon angioplasty) y con BMS o con DES de primera generación.

\section{Estudios de intervención coronaria percutánea con stents liberadores de medicamentos versus CABG}

Uno de los estudios más importantes acerca del uso de DES en enfermedad coronaria multivaso es el SYNTAX, trabajo prospectivo multicéntrico, que aleatorizó 1.800 pacientes con enfermedad del tronco principal izquierdo o de tres vasos a cirugía de bypass (CABG, por su nombre en inglés coronary artery bypass graft) o a intervención coronaria percutánea (ICP) con un stent de paclitaxel de primera generación. A un año de seguimiento, la incidencia de eventos cardíacos y cerebrovasculares adversos mayores (MACCE, sigla derivada del inglés Major Adverse Cardiac or Cerebrovascular Events) consistente en la combinación de muerte por cualquier causa, ataque cerebrovascular (ACV), infarto de miocardio (IM) o revascularización repetida, y la incidencia de revascularización repetida aislada en 1.095 pacientes del estudio que tenían enfermedad de tres vasos, fue significativamente más alta en el grupo de ICP (19,2\% vs. 11,5\%, $\mathrm{p}<0,001$, y $14,6 \%$ vs. $5,5 \%, \mathrm{p}<0,001$, respectivamente), en tanto que la tasa del punto final compuesto de muerte por todas las causas/ACV/IM fue similar con ICP y CABG $(8,0 \%$ vs. $6,6 \%, p=0,39)$. En la cohorte total la incidencia de ACV fue mayor con CABG $(2,2 \% \text { vs. } 0,6 \%, p=0,003)^{15}$. En el seguimiento final a 5 años del subgrupo de pacientes con enfermedad de tres vasos, el grupo sometido a ICP tuvo una incidencia mayor de MACCE $(37,5 \%$ vs. $24,2 \%, p<0,001)$, del punto final compuesto de muerte/ACV/IM (22,0\% vs. $14,0 \%, p<0,001)$, muerte por todas las causas $(14,6 \%$ vs. $9,2 \%, p=0,006)$, IM $(9,2 \%$ vs. $4,0 \%, p=0,001)$ y revascularización repetida $(25,4 \%$ vs. $12,6 \%, p<0,001)$; la incidencia de ACV fue similar a 5 años en ambos grupos (3,0\% vs. 3,5\%, $p=0,66$ ). Sin embargo, los resultados (de manera similar a como ocurrió en el seguimiento a un año) dependieron de la complejidad anatómica de las lesiones, definida por el sistema de puntaje. En el subgrupo de pacientes con un puntaje SYNTAX bajo (0-22) el grupo de ICP tuvo una incidencia de MACCE similar $(33,3 \%$ vs. $26,8 \%, p=0,21)$ pero mayor incidencia de revascularización repetida $(23,1 \%$ vs. $14,9 \%, p=0,038)$, mientras en los subgrupos con un puntaje SYNTAX intermedio (23-32) o alto ( $\geq 33$ ) el CABG demostró superioridad significativa en términos de MACCE, muerte, IM y revascularización repetida; las diferencias en el MACCE en favor del CABG fueron aún mayores en los diabéticos. El estudio concluyó que los resultados a cinco años de la revascularización con CABG o con ICP con un DES de paclitaxel de primera generación en pacientes con enfermedad de tres vasos, sugieren que el CABG debe permanecer como el estándar de manejo pues se traduce en tasas significativamente menores de muerte, IM y revascularización repetida, con tasas de ACV similares, aunque en pacientes con un puntaje SYNTAX bajo la ICP es una estrategia de revascularización aceptable, a expensas de tasas significativamente mayores de revascularización repetida ${ }^{16}$.

ARTS I fue un estudio aleatorizado que comparó el CABG $(n=605)$ con el uso de BMS $(n=600)$ en enfermedad coronaria multivaso, mientras que ARTS ॥ fue un estudio prospectivo multicéntrico no aleatorizado designado para comparar la seguridad y eficacia de un stent liberador de sirolimus de primera generación (SES) en pacientes con enfermedad coronaria multivaso $(n=607)$ con los brazos quirúrgico y de BMS del estudio ARTS I como controles históricos y publicó un seguimiento final a 5 años. En ARTS ॥, $46 \%$ y $54 \%$ de 
los pacientes tenían enfermedad de dos o tres vasos, respectivamente. En ARTS I, la mayoría de los pacientes tenía enfermedad de dos o tres vasos en ambos brazos del estudio $(66 \%$ y $30 \%$ respectivamente en el brazo de CABG y $69 \%$ y $27 \%$ respectivamente en el brazo sometido a BMS). A 5 años, la tasa de sobrevida libre de eventos del punto final compuesto de muerte/ACV/IM fue de $87,1 \%$ en ARTS ॥ SES comparado con $86,0 \%$ en ARTS । CABG $(p=0,1)$ y $81,9 \%$ en ARTS I BMS $(P=0.007)$. La incidencia del MACCE (compuesto de muerte de todas las causas/cualquier IM/ACV/cualquier reintervención) en ARTS ॥ SES $(27,5 \%)$ fue significativamente mayor que en ARTS I CABG $(21,1 \%, p=0,02)$ pero menor que en ARTS I BMS $(41,5 \%, p<0,001)$, impulsado en buena parte por diferencias en las tasas de revascularización repetida (libertad de revascularización a 5 años de 91,0\% en ARTS । CABG, de $79,2 \%$ en ARTS ॥ SES y de $69,1 \%$ en ARTS I BMS, con $\mathrm{p}<0,001$ en la comparación de ARTS ॥ SES con ARTS I CABG y ARTS । BMS). Un porcentaje importante (32\%) de eventos cardíacos mayores (MACE, sigla derivada del inglés major adverse cardiac events) en ARTS ॥ SES se relacionó con trombosis del stent posible, probable o definida. En ARTS ॥ SES se observó una diferencia significativa en la sobrevida libre de MACCE cuando los pacientes se estratificaron de acuerdo con el puntaje SYNTAX en riesgo bajo (SYNTAX $<16, \mathrm{n}=209$ ), intermedio (SYNTAX de 16 a 24, $\mathrm{n}=199$ ) y alto (SYNTAX > 24, $n=199$ ), a favor de los pacientes con puntaje bajo $(80,1 \%)$ en comparación con aquellos con puntaje intermedio $(70,1 \%, p=0,02)$ y alto $(67,1 \%, p=0,001)$. El estudio concluyó que en un seguimiento a cinco años la revascularización con SES tuvo una seguridad comparable a la del CABG y superior a la de los BMS y una tasa de MACCE más alta que la del CABG pero más baja que la de los BMS. Adicionalmente, se determinó que aproximadamente un tercio de los eventos adversos en los pacientes tratados con SES podrían ser prevenidos con una disminución de la trombosis del stent temprana, tardía y muy tardía, hecho importante si se tiene en cuenta el mejor desempeño, en este sentido , de los DES de segunda generación ${ }^{17}$.

CARDia fue un estudio prospectivo multicéntrico aleatorio que intentó demostrar la no inferioridad de la ICP con stent comparada con el CABG en 510 pacientes con diabetes y enfermedad coronaria multivaso ( 2 vasos $31,3 \%, 3$ vasos $61,7 \%$ ) o enfermedad de un vaso compleja (origen o tercio proximal de la coronaria descendente anterior, $7 \%$ ) sintomáticas. Inicialmente se utilizaron BMS pero se hizo un cambio al stent Cypher ${ }^{\circledR}$ (liberador de sirolimus de primera generación) cuando estos stents estuvieron disponibles (69\% del total de los pacientes). A un año de seguimiento, la incidencia del punto final compuesto de muerte/IM/ACV fue de $10,5 \%$ en el grupo de CABG y de $13,0 \%$ en el grupo de ICP $(p=0,39)$, la mortalidad por todas las causas fue de $3,2 \%$ para cada una, en tanto que la del punto final compuesto de muerte/IM/ACV/revascularización repetida fue de $11,3 \%$ y $19,3 \%(p=0,02)$, respectivamente. Al comparar los pacientes sometidos a CABG con aquellos que recibieron stent Cypher ${ }^{\circledR}$ la incidencia del punto final compuesto de muerte/IM/ACV fue de $12,4 \%$ y $11,6 \%$ $(p=0,82)$ respectivamente. En el estudio no se alcanzó el margen de no inferioridad predeterminado pues hubo una incidencia mayor (aunque no significativa) del punto final compuesto de muerte/IM/ACV (impulsada por una tasa más alta de IM) y una incidencia significativamente mayor de revascularización repetida en el grupo de ICP. Sin embargo, las tasas de ACV mostraron una tendencia en favor de la ICP y la mortalidad fue igual en ambos grupos ${ }^{18}$.

FREEDOM fue un estudio prospectivo multicéntrico aleatorizado que comparó la ICP con DES de primera generación (Cypher ${ }^{\circledR}$ en $51 \%$ o Taxus ${ }^{\circledR}$ en $43 \%$, en quienes finalmente fueron sometidos a ICP) con el CABG en 1.900 pacientes con diabetes y enfermedad coronaria de dos o tres vasos $(17 \%$ y $83 \%$ de los pacientes, respectivamente). El puntaje SYNTAX promedio fue de $26,2 \pm 8,6$ ( $<22$ en $35 \%$, 22-32 en $45 \%$ y $\geq 33$ en $20 \%$ de los pacientes). A 30 días el punto final primario de muerte de cualquier causa/IM no fatal/ACV no fatal había ocurrido en un menor número de pacientes en el grupo de ICP (26 vs. 42 ), pero las curvas comenzaron a separarse en favor del CABG a los dos años de seguimiento y a cinco años la incidencia fue de $26,6 \%$ en el grupo de ICP y de $18,7 \%$ en el grupo de CABG ( $p=0,005)$, con el beneficio del CABG impulsado por diferencias en las tasas de IM $(13,9 \%$ vs. $6,0 \%, p<0,001)$ y de muerte por cualquier causa $(16,3 \%$ vs. $10,9 \%, p=0,049)$. El grupo de ICP presentó menos ACV a 5 años (2,4\% vs. 5,2\%, $p=0,03)$, con mayoría del exceso de ACV en el grupo de CABG en los primeros 30 días luego de la aleatorización, pero a un año tuvo mayor incidencia de revascularización repetida $(12,6 \%$ vs. $4,8 \%, p<0,001)$. Se concluyó que el CABG fue superior a la ICP con DES de primera generación en pacientes con diabetes y enfermedad coronaria multivaso (predominantemente tres vasos) por una disminución significativa en las tasas de muerte e IM, aunque con mayor incidencia de $\mathrm{ACV}^{19}$.

De otro lado, en ERACI III, un estudio multicéntrico prospectivo no aleatorizado, 225 pacientes con enfermedad multivaso (dos vasos en $61,8 \%$ y tres vasos en $38,2 \%$ ) que recibieron DES de primera generación (SES o PES) y que cumplieron los criterios de inclusión clínicos y angiográficos del estudio ERACI II fueron comparados con los dos brazos de tratamiento (ERACI ॥-BMS y ERACI ॥-CABG, $n=225$ en cada brazo) de este último estudio. A un año de seguimiento la libertad de MACCE (definida como ausencia de la combinación de muerte por cualquier causa/IM no fatal/ACV mayor/revascularización repetida) fue significativamente mayor en la cohorte ERACI III-DES (88\%) que en la cohorte ERACI ॥-CABG $(80,5 \%, p=0,01)$ y en la cohorte ERACI ॥-BMS $(77,7 \%, p=0,01)$. La cohorte ERACI III-DES tuvo una libertad de muerte/IM similar a la cohorte ERACI ॥-BMS (94,3\% y $94,6 \%$ respectivamente, $p=N S$ ) y en ambas fue mayor que la de la cohorte ERACI ॥-CABG $(86,2 \%, p=0,01$ para ambas comparaciones). La libertad de revascularización repetida fue similar en ERACI III-DES y ERACI ॥-CABG (91,2\% y 94,7\% respectivamente, $\mathrm{p}=\mathrm{NS}$ ) y ambas fueron significativamente mejores que la de ERACI $॥$-BMS (83\%, $p<0,001$ para ambas comparaciones) , se concluyó que los pacientes con enfermedad multivaso tratados con DES de primera generación en ERACI III tuvieron mejores desenlaces que aquellos tratados con BMS o CABG en ERACI ॥. La tasa de MACCE a tres años fue menor en ERACI II-DES (22,7\%) que en ERACI ॥-BMS $(29,8 \%, p=0,016)$, debido principalmente a una disminución sostenida en la revascularización repetida (14,2\% vs. $24,4 \%$, $\mathrm{p}=0,008)$. Aunque a un año los datos clínicos indicaron una ventaja significativa de ERACI ॥-DES sobre ERACI ॥-CABG, las tasas de MACCE convergieron a los tres años $(22,7 \%$ en ambas cohortes), al parecer por un mayor requerimiento tardío (luego de un año) de revascularización repetida $(5,2 \%$ 
vs. $0,9 \%, p=0,012$ ) y una tendencia hacia un incremento tardío en la incidencia de la combinación de muerte/IM no fatales $(4,4 \%$ vs. $2,2 \%, p=0,29)$ en la cohorte ERACI $॥-D E S$. La ventaja de los DES sobre los BMS en términos de MACCE vista a un año, se estrechó posteriormente pero permaneció significativa a tres años. Se presentaron 9 casos de trombosis del stent subaguda, tardía o muy tardía (>30 días) en el grupo de DES y ninguna en el grupo de BMS $(p=0,008)^{20,21}$.

BEST fue un estudio prospectivo multicéntrico aleatorio de no inferioridad diseñado para comparar los desenlaces en pacientes con enfermedad coronaria de dos o tres vasos tratados con ICP con un DES de segunda generación (stent liberador de everolimus, EES por su nombre en inglés everolimus-eluting stent) o con CABG. Los autores planearon aleatorizar 1.776 pacientes pero el estudio fue suspendido de manera prematura cuando se habían incluido solo 880 pacientes por una aleatorización muy lenta (enfermedad de dos vasos en $22,8 \%$ y de tres vasos en $77,2 \%$ de los pacientes). El punto final primario fue un compuesto de muerte/IM/revascularización repetida a dos años, pero también se analizaron los eventos en un seguimiento a más largo plazo (mediana de 4,6 años). A los dos años, el punto final primario había ocurrido en $11,0 \%$ de los pacientes en el grupo de ICP y en $7,9 \%$ en el grupo de CABG $(p=0,32$ para no inferioridad). En el seguimiento a largo plazo, el punto final primario había ocurrido en $15,3 \%$ en el grupo de ICP y en $10,6 \%$ en el grupo de CABG $(p=0,04)$ y aunque no hubo diferencia significativa en un compuesto de muerte/IM/ACV $(11,9 \%$ vs. $9,5 \%, p=0,26)$, las tasas de revascularización repetida $(11,0 \%$ vs. $5,4 \%, p=0,003)$ y de IM espontáneo $(4,3 \%$ vs. $1,6 \%, p=0,02)$ fueron significativamente más altas en el grupo de ICP. Los autores concluyeron que en pacientes con enfermedad multivaso, la tasa de eventos cardíacos mayores adversos fueron más altas en aquellos sometidos a ICP con EES que en aquellos sometidos a CABG. Sin embargo, la terminación prematura del estudio disminuyó su poder estadístico ${ }^{22}$.

\section{Metaanálisis}

Se han publicado al menos tres metaanálisis de estudios que comparan la ICP con DES de primera generación con el CABG en pacientes con enfermedad multivaso ${ }^{23-25}$. En general, concluyen que aunque la ICP con DES es segura comparada con el CABG, las tasas de MACCE son más altas con la ICP debido principalmente a un exceso de revascularización repetida, si bien con el CABG hay un aumento en las tasas de ACV.

\section{Registros}

Dos estudios compararon los desenlaces en pacientes con enfermedad coronaria de dos o tres vasos sometidos a ICP con DES de primera generación o a CABG utilizando las bases de datos CSRS (Cardiac Surgery Reporting System) y PCIRS (Percutaneous Coronary Intervention Reporting System) del Departamento de Salud del Estado de Nueva York, las cuales recolectan la información de todos los residentes de dicho estado sometidos a CABG O ICP. El primer estudio incluyó los pacientes tratados entre octubre $1^{\circ}$. de 2003 y diciembre 31 de $2004^{26}$ y el segundo los pacientes tratados entre octubre $1^{\circ}$. de 2003 y diciembre 31 de $2005^{27}$; adicionalmente en este último los autores realizaron un análisis de propensión que igualó 8.121 parejas de pacientes sometidos a ICP con DES o a CABG, para controlar el sesgo de selección del tratamiento. En ambos estudios se encontró que el CABG se asoció con menor riesgo de muerte que la ICP con DES en pacientes con enfermedad multivaso, aunque en el estudio más reciente esto no fue así en el subgrupo con enfermedad de dos vasos sin compromiso de la descendente anterior. En el primer estudio el CABG también se asoció con menores tasas del combinado de muerte/IM y revascularización repetida.

Cuatro estudios analizaron los desenlaces en seguimientos que fluctuaron entre uno y 5,6 años en pacientes con enfermedad multivaso tratados con ICP con DES de primera generación o CABG utilizando las bases de datos de centros únicos ${ }^{28-30,35}$. En dos de estos estudios la mayoría de los pacientes con enfermedad de dos vasos fueron sometidos a ICP con DES y la mayoría de aquellos con enfermedad de tres vasos a $C A B G^{28,29}$; ambos estudios encontraron que la ICP se asoció con mayores tasas de MACCE (incluyendo muerte, IM y revascularización repetida), aunque esta diferencia fue más evidente en los pacientes diabéticos en uno de los estudios. En los otros dos estudios la ICP con DES tuvo una mortalidad similar a la del CABG a expensas de una mayor revascularización repetida, aunque en uno de los estudios la mortalidad fue menor con los DES en los pacientes con enfermedad de dos vasos sin compromiso de la descendente anterior proximal $^{30}$ mientras que en el otro estudio se notó un beneficio de la ICP sobre el CABG en los pacientes con enfermedad de dos vasos ${ }^{35}$.

Se han publicado varios análisis de pacientes con enfermedad multivaso sometidos a ICP con EES de segunda generación o a CABG entre enero $1^{\circ}$. de 2008 y diciembre 31 de 2011 e incluidos en los registros CSRS (Cardiac Surgery Reporting System) y PCIRS (Percutaneous Coronary Intervention Reporting System) del Departamento de Salud del estado de Nueva York. En todos los estudios se analizaron cohortes con características basales similares igualadas con puntajes de propensión y los diferentes estudios incluyeron la población global de pacientes ${ }^{31}$, diabéticos ${ }^{32}$, con enfermedad renal crónica ${ }^{33}$ y con disfunción ventricular izquierda (vi) sistólica severa, definida como una fracción de expulsión $(\mathrm{FE}) \leq 35 \%^{34}$. En el análisis de la población global de pacientes se concluyó que en un seguimiento promedio de 2,9 años, el riesgo de muerte asociado con la ICP con EES fue similar al asociado con el CABG. La ICP se relacionó con un riesgo mayor de IM (entre los pacientes con revascularización incompleta) y de revascularización repetida pero con un riesgo menor de $\mathrm{ACV}^{31}$. En los pacientes diabéticos la ICP con EES se asoció con menor riesgo de muerte y de ACV a corto plazo (30 días). Sin embargo, a largo plazo (hasta 4 años) la ICP con EES se asoció con un riesgo similar de muerte, un riesgo mayor de IM (en los pacientes con revascularización incompleta) y de revascularización repetida y un riesgo menor de $\mathrm{ACV}^{32}$. En los pacientes con enfermedad renal crónica el CABG se asoció con mayor riesgo a 30 días de muerte, ACV y revascularización repetida, mientras que la ICP con EES se asoció con riesgo mayor a largo plazo (promedio de 2,9 años) de revascularización repetida y posiblemente de IM, sin diferencias significativas en la mortalidad a largo plazo. En el subgrupo de pacientes en hemodiálisis los 
resultados favorecieron al CABG sobre la ICP ${ }^{33}$. En la población con disfunción VI sistólica severa la ICP con EES tuvo una sobrevida a largo plazo (promedio de 2,9 años) comparable con la obtenida con el CABG. La ICP se asoció con mayor riesgo de IM (en los pacientes con revascularización incompleta) pero con menor riesgo de $\mathrm{ACV}^{34}$.

Un estudio de observación en pacientes con edad $\geq 65$ años y sin infarto agudo de miocardio analizó los datos de 103.549 pacientes sometidos a ICP $(78 \%$ con DES de primera generación, $16 \%$ con BMS y $6 \%$ con balón) y 86.244 pacientes sometidos a CABG por enfermedad coronaria de dos o tres vasos e incluidos en la base de datos de ICP de la American College of Cardiology Foundation (CathPCI Registry) y la base de datos de cirugía de adultos de la Society of Thoracic Surgeons (ACD) entre enero $1^{\circ}$. de 2004 y diciembre 31 de 2007. El estudio concluyó que a 4 años de seguimiento hubo una ventaja en la sobrevida entre quienes fueron sometidos a CABG comparado con aquellos sometidos a ICP. ${ }^{36}$

Finalmente, un estudio analizó el seguimiento a tres años de 2.981 pacientes con enfermedad coronaria de tres vasos incluidos en el registro multicéntrico CREDO-Kyoto $\mathrm{PCI} / \mathrm{CABG}$ registry cohort-2, de pacientes sometidos a una primera revascularización coronaria entre enero de 2005 y diciembre de 2007. De 2.981 pacientes, 1.825 fueron sometidos a ICP (se implantaron stents en $95 \%$ de los pacientes y se usó al menos un DES en 77\%, SES en $94 \%$ de ellos) y 1.125 a CABG. La ICP se asoció con un riesgo significativamente mayor de muerte/IM/ACV, de muerte por todas las causas (sin diferencia en el riesgo de muerte cardíaca), revascularización repetida e IM, sin diferencia significativa en el riesgo de $\mathrm{ACV}^{37}$.

\section{Otra evidencia}

Sripal Bangalore et al. efectuaron una búsqueda sistemática de estudios aleatorizados que compararan las diferentes modalidades de revascularización (ICP o CABG o comparación de diferentes DES) en pacientes con diabetes, para lo cual definieron nueve grupos: CABG, POBA (plain old balloon angioplasty), BMS, SES, PES, ZES-endeavor (ZES: stent liberador de zotarolimus, por su nombre en inglés zotarolimus-eluting stent), ZES-resolute (ZES-R), EES platino-cromo y CrCo (cromo-cobalto) EES. Se incluyeron finalmente 68 estudios aleatorizados con 24.015 pacientes seguidos por una media de tres años (rango, 1-10 años) para un total de 71.595 pacientes-años de seguimiento. El porcentaje de pacientes con enfermedad coronaria de dos o tres vasos varió entre los estudios. Cuatro estudios compararon CABG con POBA (4.091 pacientes-años), 5 estudios compararon CABG con BMS (4.646 pacientes-años), cinco estudios compararon CABG con DES de primera generación (13.733 pacientes-años) y el resto de los estudios compararon diferentes stents (46.959 pacientes-años). Comparadas con el CABG (RR de referencia: 1,0), las ICP con PES (RR 1,57, 95\% IC $1,15-2,19)$ o con SES (RR 1,43, 95\% IC 1,06-1,97) se asociaron con un aumento en la mortalidad. Sin embargo, las ICP con DES de segunda generación como el CrCo EES (RR $1,11,95 \%$ IC $0,57-1,84$ ) y el ZES-R (RR 1,45, 95\% IC 0,31$8,81)$ no se asociaron con un incremento significativo en la mortalidad. El punto estimado para IM para los BMS, los SES y los PES favoreció al CABG, aunque ninguno de ellos fue estadísticamente significativo. No obstante, el punto estimado para los DES de segunda generación como el $\mathrm{CrCo}$ EES y el ZES-R fue cercano a 1.0. Comparado con el CABG hubo un exceso de revascularización repetida con la ICP, la cual declinó progresivamente desde la POBA $(341 \%$ de incremento), al BMS (218\% de incremento), al PES (81\% de incremento) y al SES (47\% de incremento). Sin embargo, para la ICP con CrCo EES el incremento en la revascularización repetida no fue estadísticamente significativo (RR 1,32, 95\% IC 0,74-2,29), aunque el punto estimado favoreció al CABG, el cual se asoció con una incidencia de ACV numéricamente mayor. El estudio concluyó que en pacientes con diabetes la evidencia actual derivada de comparaciones indirectas muestra una mortalidad similar entre el CABG y la $\mathrm{PCI}$ con DES de segunda generación, principalmente con el CrCo EES. El CABG se asoció con un incremento numérico en la incidencia de ACV, en tanto que el CrCo EES con un incremento numérico en la revascularización repetida ${ }^{38}$.

\section{Análisis de la evidencia y recomendaciones}

La evidencia disponible en la revascularización de pacientes con enfermedad multivaso (dos o tres vasos) ha comparado el CABG con la POBA, con los BMS y en el mejor de los casos con DES de primera generación $\left(\right.$ Cypher $^{\circledR}$ y Taxus $^{\circledR}$ ), pues la evidencia con los DES de segunda generación es aún muy limitada y en su mayor parte indirecta. Aunque existen algunos estudios prospectivos que han comparado el CABG con la ICP con DES, algunos de ellos no han sido aleatorizados, y buena parte de la evidencia se basa en análisis retrospectivos de registros y en meta-análisis. Esta evidencia ha demostrado que en pacientes con enfermedad multivaso el CABG usualmente tiene menores tasas de mortalidad, IM y revascularización repetida a largo plazo, a expensas de al menos una tendencia a una mayor incidencia de ACV comparado con la ICP. Estas diferencias en favor del CABG aumentan a medida que se incrementa la complejidad de la enfermedad, definida por un sistema de puntaje como el SYNTAX. La diferencia en favor del CABG es más significativa con puntajes SYNTAX intermedio (23-32) y alto $(\geq 33)$, pero puede desaparecer o incluso estar en favor de la ICP en los pacientes con un puntaje SYNTAX bajo (0-22), principalmente en enfermedad de dos vasos sin compromiso de la descendente anterior proximal y en lo que respecta a la incidencia de mortalidad y de IM, puesto que la necesidad de revascularización repetida usualmente es significativamente más alta con la ICP. Las diferencias en favor del CABG son aún mayores en los pacientes diabéticos. Son varias las razones que explican la ventaja del CABG en la enfermedad multivaso: las altas tasas de permeabilidad de los injertos de arteria mamaria interna; el hecho de que los stents tratan únicamente los segmentos en los cuales son implantados mientras que los injertos quirúrgicos tienden a proteger todos los segmentos proximales a los mismos, incluidas las placas vulnerables que no han producido estenosis significativas, y el hecho de que la revascularización completa es más frecuente con el CABG que con la ICP. Por otra parte, los avances en los stents de segunda generación con struts más delgados y polímeros más biocompatibles o biodegradables o incluso con ausencia de polímeros, han mejorado sustancialmente su seguridad y eficacia, condición que ha 
llevado a estrechar potencialmente la brecha entre el CABG y la ICP y, que a su vez, hace que los estudios disponibles y las guías de manejo derivadas de los mismos para orientar las decisiones sobre la estrategia de revascularización más adecuada no estén a la par con los últimos avances tecnológicos. Por otra parte, es preciso tener en cuenta que los pacientes incluidos en los estudios aleatorizados son un subgrupo muy selecto de la totalidad de los pacientes vistos usualmente en la práctica clínica. Con frecuencia, los pacientes con enfermedad coronaria multivaso en lo que se denomina el "mundo real" son extremadamente complejos y no cumplen los criterios de inclusión y exclusión de los estudios, de modo que los resultados de los ensayos clínicos disponibles no serían aplicables en un alto porcentaje de pacientes $^{38-40}$.

Con base en la evidencia descrita, que es claramente limitada y anticuada respecto a los avances más recientes, las guías más recientes de revascularización miocárdica, publicadas por la European Society of Cardiology y la European Association of Cardio-Thoracic Surgery, hacen las siguientes recomendaciones: en enfermedad de uno o dos vasos sin compromiso de la descendente anterior proximal el CABG como recomendación IIb con nivel de evidencia C y la ICP como recomendación I con nivel de evidencia C; en enfermedad de dos vasos con compromiso de la descendente anterior proximal el CABG como recomendación । con nivel de evidencia $B$ y la ICP como recomendación I con nivel de evidencia $C$; en enfermedad de tres vasos con un puntaje SYNTAX $\leq 22$ el CABG como recomendación । con nivel de evidencia A y la ICP como recomendación I con nivel de evidencia B; y en enfermedad coronaria de 3 vasos con puntajes SYNTAX de 23 a 32 o > de 32 el CABG como recomendación । con nivel de evidencia A y la ICP como recomendación III con nivel de evidencia $B^{41}$.

En resumen, todo lo que se expone en este documento y de acuerdo con los constantes avances en tecnología, medicamentos, técnica y experiencia frecuentemente no alcanza a ser reflejado en las guías de manejo publicadas por las sociedades científicas. Por tanto, teniendo en cuenta que al hacer referencia a la ICP en la actualidad se habla de revascularización con DES de segunda generación, puede concluirse que si la anatomía coronaria es compleja (puntaje SYNTAX intermedio o alto) se debe preferir en general el CABG, pero si el paciente tiene comorbilidades que harían que el CABG fuera de alto riesgo o si el paciente no acepta la cirugía, se debe considerar la angioplastia. Para pacientes con lesiones muy localizadas que requerirían menor número de stents y stents más cortos (puntaje SYNTAX bajo) se preferirá la ICP por mayor equivalencia en desenlaces tales como mortalidad e IM y clara disminución en la necesidad de revascularización repetida, que es un desenlace inherente mayor con la ICP. Lo precedente es aún más cierto en los pacientes con enfermedad de dos vasos sin compromiso de la descendente anterior proximal, en la que la ICP tiende a aventajar al CABG en todos los desenlaces. Este último es la estrategia de revascularización predilecta en la mayoría de pacientes diabéticos con enfermedad multivaso a no ser que la anatomía sea muy favorable para la ICP, las comorbilidades o la "fragilidad" incrementen significativamente el riesgo quirúrgico o el paciente no acepte la cirugía. Otro factor a tener en cuenta es la edad, principalmente cuando tanto el CABG como la ICP son opciones igualmente factibles desde el punto de vista técnico. Debido a que los injertos venosos tienden a ocluirse con el tiempo, los problemas clínicos derivados de la enfermedad de los mismos ocurren comúnmente en los pacientes sometidos a cirugía a edades tempranas. Con el uso de la ICP como la primera opción en los pacientes jóvenes se retardan o se evitan los problemas de los injertos venosos y la cirugía se reserva como para su uso a una edad más avanzada. En quienes la anatomía coronaria y otros factores suponen una equivalencia clínica entre ambas opciones de revascularización, se realiza un análisis detallado de las mismas con el paciente. En estos casos es especialmente útil el "equipo cardíaco" (heart team), que incluye discusiones con miembros de los equipos de Cardiología intervencionista y Cirugía cardíaca, para asegurar que el paciente entiende las ventajas y desventajas de cada una de las dos estrategias de revascularización. De esta manera, cuando los desenlaces de ambas modalidades podrían ser potencialmente similares, particularmente en lo que respecta a puntos finales "duros" como mortalidad e IM, la preferencia del paciente se convierte en la piedra angular de la estrategia de manejo más apropiada. Algunos pacientes preferirán someterse a la ICP en vista de su menor morbilidad a corto plazo y asumir el riesgo potencialmente mayor de una revascularización repetida, mientras que otros preferirán someterse al CABG con miras a tener una revascularización más "durable"', pese a su mayor morbilidad inicial.

\section{Conflictos de interés}

Ninguno.

\section{Bibliografía}

1. Stettler C, Wandel S, Allemann S, et al. Outcomes associated with drug-eluting and bare-metal stents: a collaborative network meta-analysis. Lancet. 2007;370:937-48.

2. Weisz G, Leon MB, Holmes DR Jr, et al. Five-year follow-up after sirolimus eluting stent implantation results of the SIRIUS (Sirolimus- Eluting Stent in De-Novo Native Coronary Lesions) trial. J Am Coll Cardiol. 2009;53:1488-97.

3. Morice MC, Serruys PW, Barragan P, et al. Long-term clinical outcomes with sirolimus-eluting coronary stents: five-year results of the RAVEL trial. J Am Coll Cardiol. 2007;50: 1299-304.

4. Grube E, Dawkins K, Guagliumi G, et al. TAXUS VI final 5-year results: a multicentre, randomised trial comparing polymerbased moderate-release paclitaxel-eluting stent with a bare metal stent for treatment of long, complex coronary artery lesions. Eurolntervention. 2009;4:572-7.

5. Daemen J, Wenaweser P, Tsuchida K, et al. Early and late coronary stent thrombosis of sirolimus-eluting and paclitaxeleluting stents in routine clinical practice: data from a large two-institutional cohort study. Lancet. 2007;369:667-78.

6. Windecker S, Meier B. Late coronary stent thrombosis. Circulation. 2007;116:1952-65.

7. Serruys PW, Daemen J. Late stent thrombosis: a nuisance in both bare metal and drug-eluting stents. Circulation. 2007;115:1433-9.

8. Carmenzind E, Steg PG, Wijns W. Stent thrombosis late after implantation of first-generation drug-eluting stents: a cause of concern. Circulation. 2007;115:1440-5. 
9. Palmerini T, Biondi-Zoccai G, Della Riva D, et al. Stent thrombosis with drug-eluting and bare-metal stents: evidence from a comprehensive network meta-analysis. Lancet. 2012;379:1393-402.

10. Tada T, Byrne RA, Simunovic I, et al. Risk of stent thrombosis among bare-metal stents, first-generation drug-eluting stents, and second-generation drug-eluting stents: results from a registry of 18,334 patients. J Am Coll Cardiol. 2013;6:1267-74.

11. Philip F, Agarwal S, Bunte MC, Goel SS, Tuzcu EM, Ellis S, et al. Stent thrombosis with second-generation drug-eluting stents compared with bare-metal stents: network metaanalysis of primary percutaneous coronary intervention trials in ST-segment-elevation myocardial infarction. Circ Cardiovasc Interv. 2014;7:49-61.

12. Bangalore S, Toklu B, Amoroso N, et al. Bare metal stents, durable polymer drug eluting stents, and biodegradable polymer drug eluting stents for coronary artery disease: mixed treatment comparison meta-analysis. BMJ. 2013;347:f6625.

13. Navarese EP, Tandjung K, Claessen B, et al. Safety and efficacy outcomes of first and second generation durable polymer drug eluting stents and biodegradable polymer biolimus eluting stents in clinical practice: comprehensive network metaanalysis. BMJ. 2013;347:f6530.

14. Claessen BE, Henriques JPS, Jaffer FA. Stent thrombosis: a clinical perspective. J Am Coll Cardiol. 2014;7:1081-92.

15. Serruys PW, Morice M, Kappetein AP, et al. for the SYNTAX investigators. Percutaneous coronary intervention versus coronary-artery bypass grafting for severe coronary artery disease. N Engl J Med. 2009;360:961-72.

16. Head SJ, Davierwala PM, Serruys PW, et al. Coronary artery bypass grafting vs. percutaneous coronary intervention for patients with three-vessel disease: final five year follow-up of the SYNTAX trial. Eur Heart J. 2014;35:2821-30.

17. Serruys PW, Onuma $Y$, Garg $S$, et al., on behalf of the ARTS II Investigators. 5-Year clinical outcomes of the ARTS II (Arterial Revascularization Therapies Study II) of the sirolimuseluting stent in the treatment of patients with multivessel de novo coronary artery lesions. J Am Coll Cardiol. 2010;55: 1093-101.

18. Kapur A, Hall RJ, Malik IS, et al. Randomized comparison of percutaneous coronary intervention with coronary artery bypass grafting in diabetic patients: 1 -year results of the CARDia (Coronary Artery Revascularization in Diabetes) trial. J Am Coll Cardiol. 2010;55:432-40.

19. Farkouh ME, Domanski M, Sleeper LA, et al. for the FREEDOM trial investigators Strategies for multivessel revascularization in patients with diabetes. N Engl J Med. 2012;367:2375-84.

20. Rodriguez AE, Grinfeld L, Fernandez-Pereira C, et al. Revascularization strategies of coronary multiple vessel disease in the drug eluting stent era: one year follow-up results of the ERACI III trial. Eurolnterv. 2006;2:53-60.

21. Rodriguez AE, Maree AO, Mieres J, et al. Late loss of early benefit from drug-eluting stents when compared with bare metal stents and coronary artery bypass surgery: 3 years follow-up of the ERACI III registry. Eur Heart J. 2007;28:2118-25.

22. Park S, Ahn J, Kim Y, et al. for the BEST trial investigators. Trial of everolimus-eluting stent or bypass surgery for coronary disease. N Engl J Med. 2015;372:1204-12.

23. From AM, Al Badarin FJ, Cha SS, et al. Percutaneous coronary intervention with drug-eluting stents versus coronary artery bypass surgery for multivessel coronary artery disease: a metaanalysis of data from the ARTS II, CARDia. ERACI III, and SYNTAX studies and systematic review of observational data. Eurolnterv. 2010;6:269-76.

24. Al Ali J, Franck C, Filion KB, et al. Coronary artery bypass graft surgery versus percutaneous coronary intervention with first-generation drug-eluting stents: a meta-analysis of randomized controlled trials. J Am Coll Cardiol Intv. 2014;7:497-506.

25. Benedetto H, Melina G, Angeloni E, et al. Coronary artery bypass grafting versus drug-eluting stents in multivessel coronary disease: a meta-analysis on 24.268 patients. Eur J Cardiothorac Surg. 2009;36:611-5.

26. Hannan EL, Wu C, Walford G, et al. Drug eluting stent vs. coronary-artery bypass grafting in multivessel coronary disease. N Engl J Med. 2008;358:331-41.

27. Wu C, Camacho FT, Zhao S, et al. Long-term mortality of coronary artery bypass graft surgery and stenting with drug-eluting stents. Ann Thorac Surg. 2013;95:1297-305.

28. Li Y, Zheng Z, Xu B, et al. Comparison of drug-eluting stents and coronary artery bypass surgery for the treatment of multivessel coronary disease: three-year follow-up results from a single institution. Circulation. 2009;119:2040-50.

29. Javaid A, Steinberg DH, Buch AH, et al. Outcomes of coronary artery bypass grafting versus percutaneous coronary intervention with drug-eluting stents for patients with multivessel coronary artery disease. Circulation. 2007;116 suppl I. I-200-I-6.

30. Park DW, Yung SC, Lee SW, et al. Long-term mortality after percutaneous coronary intervention with drug-eluting stent implantation versus coronary artery bypass surgery for the treatment of multivessel coronary artery disease. Circulation. 2008;117:2079-86.

31. Bangalore S, Guo Y, Samadashvili Z, et al. Everolimus-eluting stents or bypass surgery for multivessel coronary disease. $N$ Engl J Med. 2015;372:1213-22.

32. Bangalore S, Guo Y, Samadashvili Z, et al. Everolimus eluting stents versus coronary artery bypass graft surgery for patients with diabetes mellitus and multivessel disease. Circ cardiovasc interv. 2015;8:e002626, http://dx.doi.org/10.1161/ CIRCINTERVENTIONS.115.002626.

33. Bangalore S, Guo Y, Samadashvili Z, et al. Revascularization in patients with multivessel coronary artery disease and chronic kidney disease: everolimus-eluting stents versus coronary artery bypass graft surgery. J Am Coll Cardiol. 2015;66:1209-20.

34. Bangalore S, Guo Y, Samadashvili Z, et al. Revascularization in patients with multivessel coronary artery disease and severe left ventricular systolic dysfunction: everolimus-eluting stents versus coronary artery bypass graft surgery. Circulation. 2016;133:2132-40.

35. Park DW, Kin YH, Song HG, et al. Long-term comparison of drug-eluting stents and coronary artery bypass grafting for multivessel coronary revascularization: 5-year outcomes from the Asan Medical Center-Multivessel Revascularization Registry. J Am Coll Cardiol. 2011;57:128-37.

36. Weintraub WS, Grau-Sepulveda MV, Weiss JM, et al. Comparative effectiveness of revascularization strategies. N Engl J Med. 1467;201:76.

37. Tazaki J, Shiomi H, Morimoto T, et al. Three-year outcome after percutaneous coronary intervention and coronary artery bypass grafting in patients with triple-vessel coronary artery disease: observations from the CREDO-Kyoto PCI/CABG registry cohort2. Eurolnterv. 2013;9:437-45.

38. Bangalore S, Toklu B, Feit F. Outcomes with coronary artery bypass graft surgery versus percutaneous coronary intervention for patients with diabetes mellitus: can newer generation drug-eluting stents bridge the gap? Circ cardiovasc Interv. 2014; 7:518-25.

39. Bangalore S. Applicability of the COURAGE BARI 2D, and FREEDOM trials to contemporary practice. J Am Coll Cardiol. 2016;68:996-8.

40. Kansara P, Weiss S, Weintraub WS, et al. Clinical trial versus clinical practice: when evidence and practice diverge - Should nondiabetic patients with 3-vessel disease and stable ischemic 
heart disease be preferentially treated with CABG? J Am Coll Cardiol Intv. 2015;8:1647-56.

41. Windecker S, Kolh P, Alfonso F, et al. 2014 ESC/EACTS Guidelines on myocardila revascularization: the Task Force on myocardial revascularization of the European Society of Cardiology (ESC) and the European Association for Cardio-Thoracic Surgery (EACTS). Eur Heart J. 2014;35:2541-619. 